\title{
AVALIAÇÃO DE HEPATOTOXICIDADE EM IDOSOS COM HIV/AIDS EM USO DE ISONIAZIDA
}

\section{ARTIGO ORIGINAL}

SANTANA, Claudinei Alves [1], SANTOS, Gustavo Alves Andrade dos [2]

SANTANA, Claudinei Alves. SANTOS, Gustavo Alves Andrade dos. Avaliação de hepatotoxicidade em idosos com HIV/AIDS em uso de isoniazida. Revista Científica Multidisciplinar Núcleo do Conhecimento. Ano 05, Ed. 09, Vol. 04, pp. 98107. Setembro de 2020. ISSN: 2448-0959, Link de acesso: https://www.nucleodoconhecimento.com.br/saude/hepatotoxicidade. $\quad$ DOI: 10.32749/nucleodoconhecimento.com.br/saude/hepatotoxicidade

\section{RESUMO}

Introdução: O envelhecimento da população se torna notório a partir do século $\mathrm{XX}$ alcançando vários países do mundo. Os dados do Boletim Epidemiológico de 2019 do ministério da saúde informam um aumento na incidência de casos de aids entre idosos do sexo masculino no período de 2008 a 2018 de 9,7 \% e uma redução de 5\% entre as mulheres. $\mathrm{O}$ comprometimento do sistema imunológico favorece o surgimento de doenças consideradas oportunistas sendo a Tuberculose (TB) a maior causa de morte entre os indivíduos com aids. Umas das medidas para prevenção da coinfecção TB/HIV em adultos jovens e idosos, segundo a Organização Mundial de Saúde, é o tratamento com o medicamento isoniazida, que tem por objetivo a redução do risco de adoecimento pelo Mycobacterium tuberculosis e consequentemente a manifestação da TB. Em relação a isoniazida, a hepatotoxicidade é uma reação adversa potencialmente grave que pode resultar até em morte. Objetivo: Avaliar o risco de hepatotoxicidade ao uso de isoniazida medida através dos resultados de exames laboratoriais através das enzimas hepáticas. METODO: Estudo transversal retrospectivo descritivo e analítico com pacientes idosos com HIV/aids em uso de 
isoniazida em acompanhado em Serviço Especializado em HIV/aids de 2008 a 2011. Foram analisados resultados laboratoriais de transaminases hepáticas (AST e ALT) e gama glutariltranferase de uma base de dados secundária em 3 momentos específicos (pré tratamento, concomitante ao tratamento e pós tratamento com isoniazida) e características dos sujeitos (idade, sexo, tempo de infecção pelo HIV). Os dados foram analisados em Programa Estatístico SPSS 20. Resultado: Foram analisados 12 sujeitos com idade igual ou superior a 60 anos sendo $75 \%$ (9) homens, idade média de 63 anos e 25\% (3) mulheres, idade média de 68 anos. Os sujeitos apresentavam em média 15 anos de infecção pelo HIV. Houve um aumento de 20,9 $\%$ da AST, e $11,5 \%$ da Gama Gutamiltransferase concomitante ao tratamento em relação aos valores pré tratamento. Conclusão: Os resultados laboratoriais ao uso de isoniazida apresentaram elevação indicado uma leve hepatotoxicidade sem necessidade de suspensão do tratamento preventivo com isoniazida, no entanto, acompanhamento por equipe multiprofissional e farmacêutico se faz necessário.

Descritores: AIDS, idoso, isoniazida, hepatotoxicidade, tuberculose.

\section{INTRODUÇÃO}

O envelhecimento da população se torna notório a partir do século $X X$ alcançando vários países do mundo, sendo assim os dados epidemiológicos demostram que os indivíduos ultrapassaram facilmente os 60 anos de idade no século XXI (WHO, 2015) As alterações fisiológicas em decorrência do envelhecimento aumentam a incidência e prevalência de doenças não infecciosas nessa população como hipertensão, diabetes, artrite, osteoporose. Há também a ocorrência de doenças infecciosas como Aids e Tuberculose. (RIBAS, 2014)

As comorbidades citadas apresentam um impacto negativo nos idosos pois há necessidade de intervenções farmacológicas como tratamento principal. (RIBAS, 2014)

A síndrome da imunodeficiência adquirida (AIDS) surgiu como uma das principais epidemias de doenças infecciosas do século XX (BIGGAR, 1984; COOK, 2011). A 
aids, causada pela infecção pelo vírus da imunodeficiência humana (HIV) é o resultado de um dano principalmente na imunidade mediada por células, embora haja também comprometimento da imunidade humoral. (BARASA, 2011; MOIR, 2011)

Os dados do Boletim Epidemiológico de 2019 do ministério da saúde informam um aumento na incidência de casos de aids entre idosos do sexo masculino no período de 2008 a 2018 de 9,7 \% e uma redução de 5\% entre as mulheres no mesmo período. (BRASIL, 2019)

O comprometimento do sistema imunológico favorece o surgimento de doenças consideradas oportunistas causadas por vírus, fungos e bactérias em pacientes vivendo com HIV/aids sendo a Tuberculose a maior causa de morte entre os indivíduos com aids (El-SADR, 2008; STERLING, 2010; JONG, 2004)

Em relação a Tuberculose (TB), esta trata-se de uma patologia importante clinicamente, sendo uma doença crônica, infecciosa e contagiosa, causada pelo Mycobacterium tuberculosis (Mtb), que pode atingir todos os grupos etários, porém 85\% dos casos de TB ocorrem em adultos. (SILVA, 2004).

Umas das medidas para prevenção da coinfecção TB/HIV em adultos jovens e idosos, segundo a Organização Mundial de Saúde, é o tratamento preventivo com medicamento isoniazida que tem por objetivo a redução do risco de adoecimento pelo Mtb e consequentemente a manifestação da TB. (CONDE, 2009; HORSBURGH, 2011; DUARTE, 2007).

No Brasil, a fim de reduzir o risco de TB em pacientes vivendo com HIV/aids, o Ministério da Saúde recomenda a Terapia Preventiva com Isoniazida (TPI) na dose de 5 a $10 \mathrm{mg} / \mathrm{kg} /$ dia de peso até $300 \mathrm{mg}$ por 6 meses consecutivos para o tratamento (CONDE, 2009)

A Reação Adversa ao Medicamento é definida como "qualquer efeito prejudicial ou indesejado que se manifeste após a administração do medicamento, em doses normalmente utilizadas no homem para profilaxia, diagnóstico ou tratamento de uma enfermidade. (WHO, 2002)

Em relação ao uso de isoniazida, a hepatotoxicidade, é uma reação adversa potencialmente grave que pode resultar até em morte se o medicamento não for retirado logo após o desenvolvimento de sintomas da hepatite. (CONDE, 2009; COHN, 2000; LOBUE, 2003) 
A hepatotoxicidade pode ser avaliada com a elevação assintomática de enzimas hepáticas como a AST (aspartato aminotransferase), ALT (alanina aminotransferase) e GGT (gama-glutamiltransferase). (CHURCHYARD, 2007; LOBUE, 2003)

Com o aumento da incidência de HIV/aids na população idoso e o risco de tuberculose, justifica-se a necessidade da avaliação efetiva do risco de hepatotoxicidade em idosos em tratamento preventivo com isoniazida à pacientes idosos vivendo com HIV/aids. (SILVA, 2004; LEUNG, 2011)

O profissional farmacêutico pode colaborar através de seus conhecimentos técnicos na avaliação do risco da hepatotoxicidade como a principal reação adversa presente na isoniazida no paciente idoso com HIV/aids através dos resultados laboratoriais de enzimas hepáticas. (SILVA, 2004; LALVANI, 2010)

Este artigo tem por objetivo avaliar a presença de hepatotoxicidade em pacientes idosos vivendo com HIV/aids em uso de isoniazida para prevenção de tuberculose atendidos em um Serviço Especializado entre os anos de 2008 a 2011 através de resultados de exames laboratoriais de enzimas de função hepática ALT, AST e GGT. Propõem-se também apresentar as características da população do estudo em relação a idade, sexo, tempo de infecção pelo HIV.

\section{METODOLOGIA}

Trata-se de um estudo retrospectivo, descritivo e analítico em pacientes adultos idosos com HIV/aids em seguimento no Serviço Especializando no atendimento de pacientes HIV/Aids do Hospital das Clínicas da Faculdade de Medicina da USP entre os anos de 2008 a 2011.

O presente estudo foi realizado com informações presentes em planilha excel de um banco de dado secundário, construído a partir de informações coletadas de prontuários e resultados de exames laboratórios, advindo de pesquisa anterior autorizado pelo Comitê de Ética e Pesquisa do Hospital das Clínicas da Faculdade de Medicina da Universidade de São Paulo em 2008 sob o número 1068/08.

Sendo um estudo realizado a partir de dados secundários não houve necessidade de aplicação de Termo de Consentimento Livre e Esclarecido aos pacientes. 
Foram considerados elegíveis para o estudo pacientes HIV/aids com 60 anos ou mais em uso de TPI na dose de 5 a $10 \mathrm{mg} / \mathrm{kg} /$ dia de peso até $300 \mathrm{mg}$ por até 6 meses. As analises estatísticas foram realizadas no programa estatístico SPSS - Statistics, versão 20, apresentadas as análises descritivas das variáveis de interesse na forma de medidas de tendência central (média, mediana, desvio padrão, mínima e máxima) e proporções. A análise de associação entre os valores laboratoriais dos marcadores hepáticos pré e concomitante ao uso de isoniazida foram realizadas através do teste $t$ Pareado com IC de 95\%, sendo considerado com significado estatístico $p \leq 0,05 \%$.

\section{RESULTADOS}

Foram analisados 12 pacientes com idade mínima de 60 anos e máxima de 74 anos dos quais $75 \%$ (9) eram homens (idade média de 63 anos) e $25 \%$ (3) eram mulheres (idade média de 68 anos). Os pacientes apresentavam em média 15 anos de infecção pelo HIV, variando 7 anos de mínima a 21 anos de máxima (desvio padrão 4,8 anos).

Gráfico 1. Proporção de pacientes idosos com HIV/aids em relação ao sexo

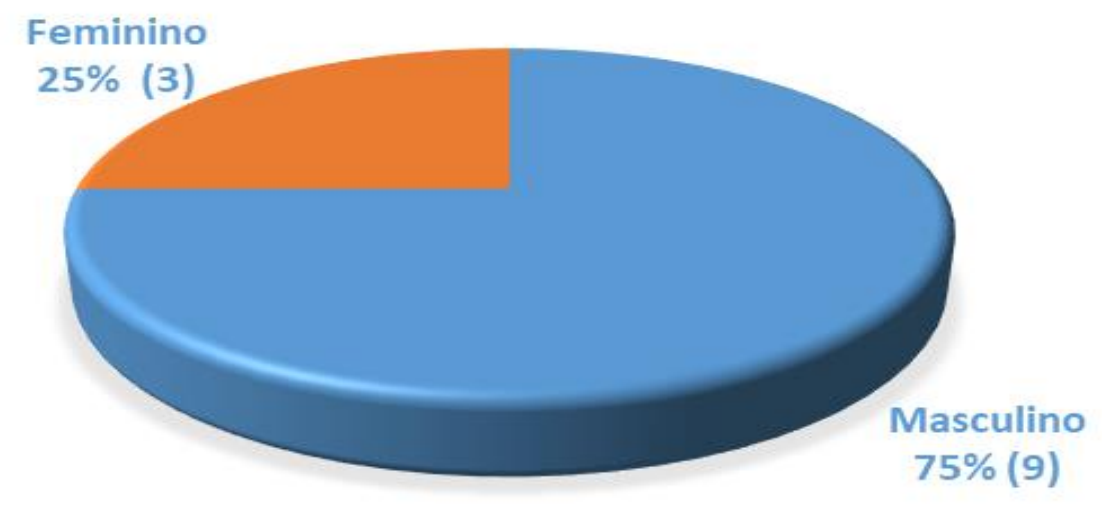

Fonte: próprio autor 
Gráfico 2. Distribuição de pacientes idosos com HIV/aids por idade

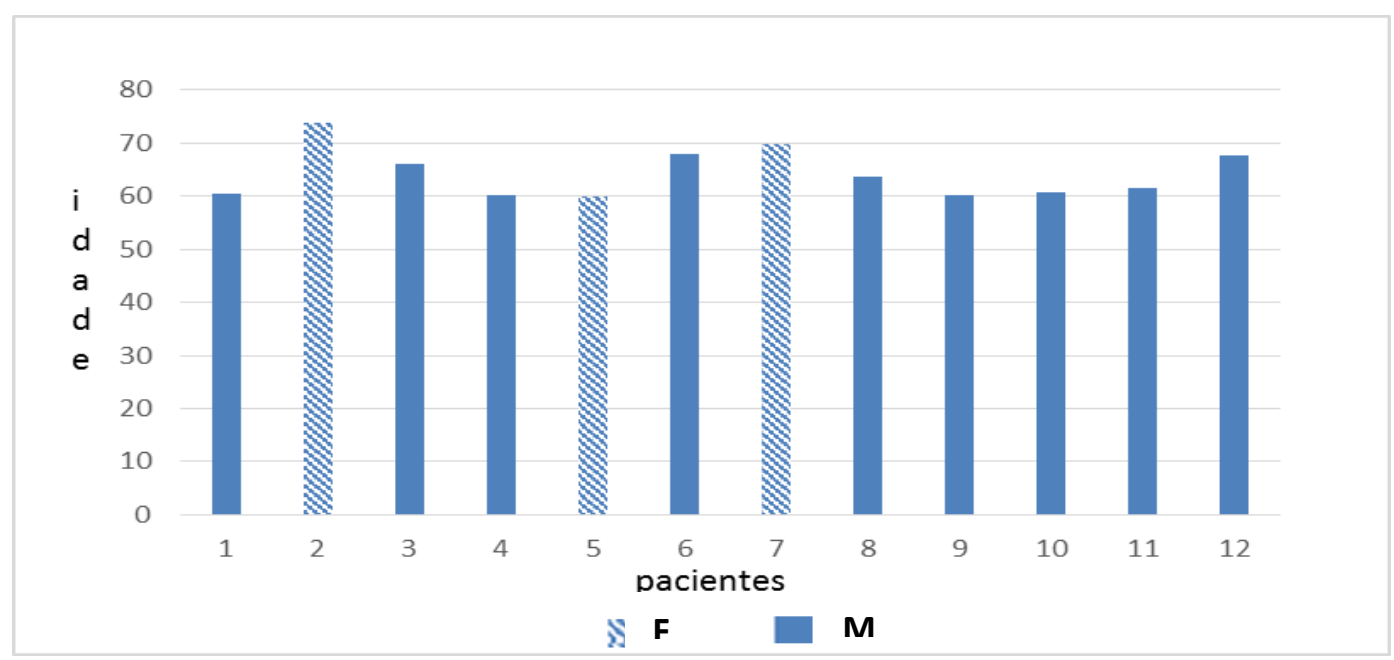

Fonte: próprio autor

Gráfico 3. Distribuição de pacientes idosos com HIV/aids por anos de infecção

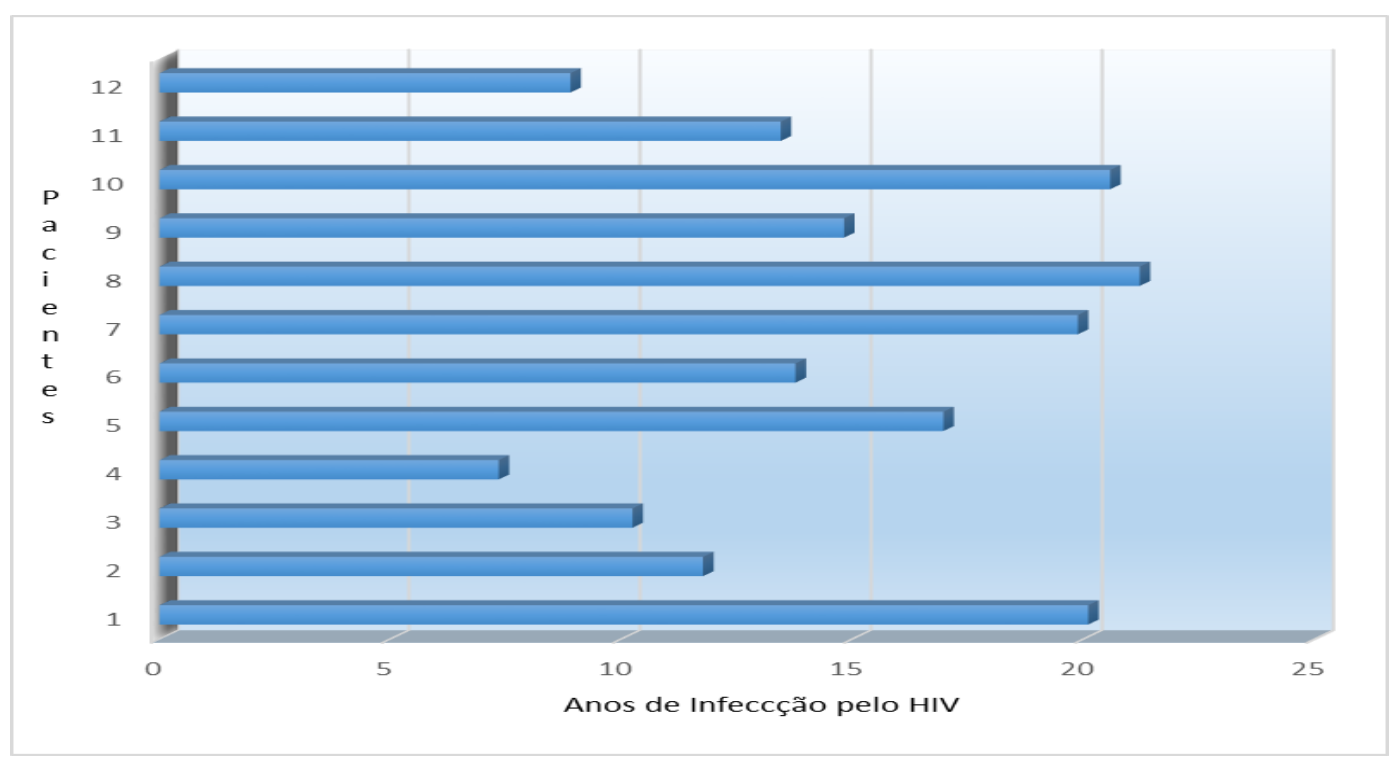

Fonte: próprio autor 
Gráfico 4. Distribuição dos resultados de exames de função hepática ALT, AST e GGT pré TPI para pacientes idosos com HIV/aids.

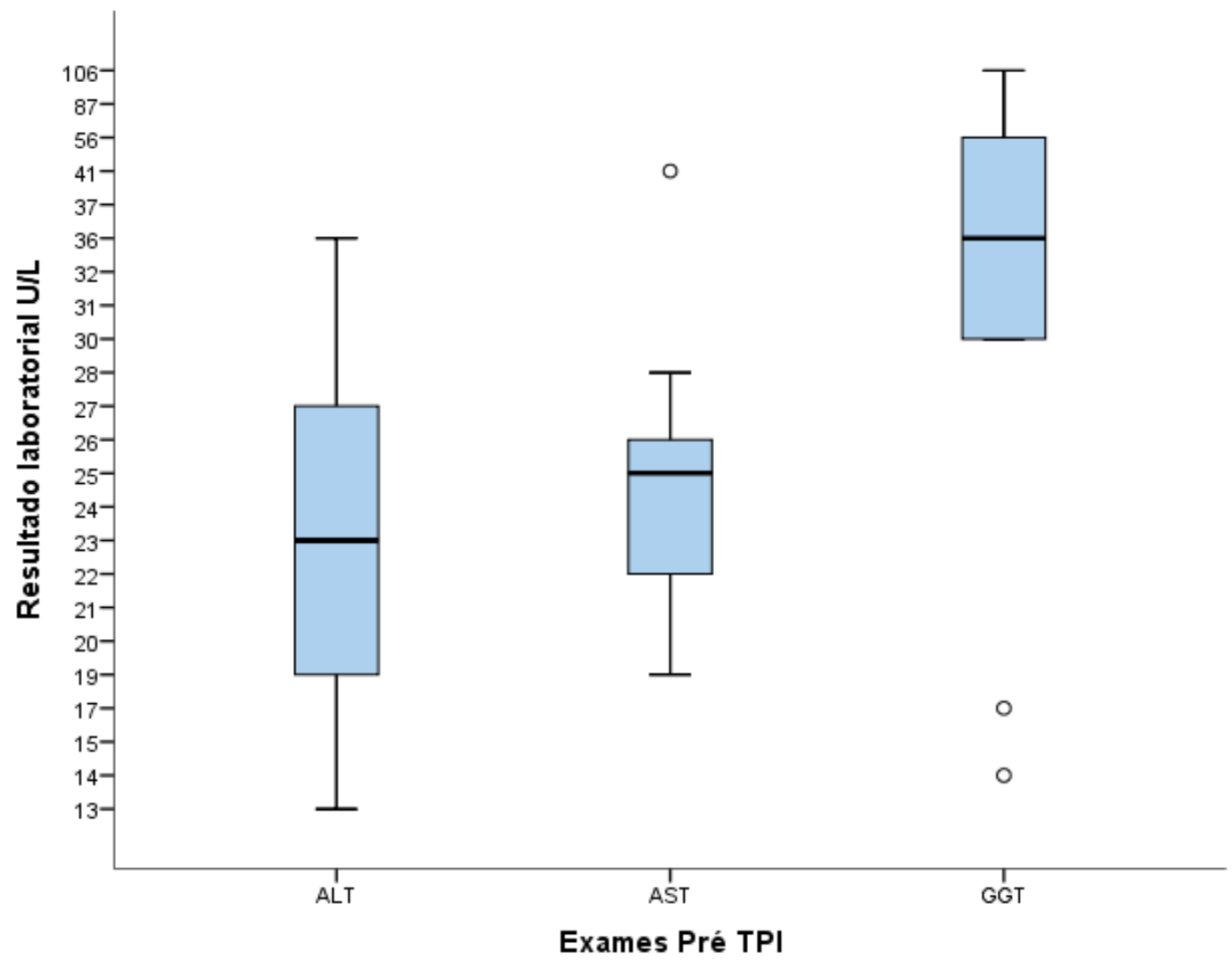

Fonte: próprio autor

Tabela 1: Mediana dos resultados de exames laboratoriais de função hepática pré, concomitante e pós TPI em pacientes idosos com HIV/aids.

\begin{tabular}{|c|c|c|c|}
\hline Período & Exames & Exames & Exames \\
\hline & AST (U/L) & ALT $(\mathrm{U} / \mathrm{L})$ & GGT (U/L) \\
\hline pré TPI & 24,5 & 23,0 & 34,5 \\
\hline concomitante TPI & 31,0 & 22,5 & 39,0 \\
\hline pós TPI & 24,5 & 22,5 & 32,0 \\
\hline
\end{tabular}

Fonte: próprio autor 
Houve elevação nos resultados laboratoriais concomitantes ao uso de TPI em 20,9 \% das enzimas AST e 11,5\% da GGT em relação aos valores pré tratamento.

Em média o resultado de AST concomitante ao TPI ( $\mathrm{M}=31,0 \mathrm{U} / \mathrm{L})$ foi superior ao resultado pré TPI $(24,5 \mathrm{U} / \mathrm{L}) \operatorname{com} \mathrm{p}=0,02$.

\section{DISCUSSÃO}

A população idosa aumenta no mundo. (WHO, 2015). No presente estudo somente 2 pacientes do sexo feminino apresentaram idade igual ou superior a 70 anos. Importante salientar que ao envelhecer também há o aumento das doenças crônicas com maior utilização de medicamentos para controle de destas condições (RIBAS, 2014). Com o aumento de pacientes idosos com HIV/aids, segundo os Boletins Epidemiológicos, haverá maior necessidade de acompanhamento por equipe multiprofissional de saúde.

Em relação ao tempo de infecção pelo HIV, 4 pacientes (3 homens e 1 mulher) apresentaram 20 anos ou mais de infecção pelo HIV. O tratamento antirretroviral apresenta reações adversas importantes que associado ao envelhecimento pode proporcionar uma piora da qualidade de vida aos pacientes. (HALLAL, 2010). O uso dos antirretrovirais e outros medicamentos para doenças crônicas ou para combater as reações adversas dos antirretrovirais podem favorecer a hepatotoxicidade. (FATIMA, 2009)

Ao utilizar o medicamento isoniazida houve um aumento das enzimas marcadoras da função hepática (AST e GGT) quando comparado com os exames laboratoriais pré tratamento caracterizando hepatotoxicidade. No entanto, o aumento não foi superior a 2 vezes o valor pré tratamento que justificasse a suspensão do uso. (CONDE, 2009) Ponto importante é que a isoniazida para o tratamento preventivo da tuberculose associada a outros medicamentos poderá apresentar-se como um fator de risco adicional para hepatotoxicidade em pacientes idosos com HIV/aids.

Diante do envelhecimento, doenças crônicas associadas e a situação do HIV/aids, os pacientes idosos podem realizar a uma intensa polifarmácia. (RAMOS, 2016) A partir desse momento as interações medicamentosas deletérias, reações adversas e intoxicações podem surgir sendo necessário a intervenção do farmacêutico junto a 
essa população com conhecimentos específicos e experiência em identificação e intervenção em Problemas Relacionados ao Medicamento.

\section{CONSIDERAÇÕES FINAIS}

A hepatotoxicidade relacionada ao uso de isoniazida esteve presente nos pacientes idosos em terapia preventiva confirmando a teoria relacionado a este medicamento. Contudo não houve suspensão do tratamento, porém, diante da utilização da polifarmácia se faz necessário o acompanhamento do paciente pela equipe multiprofissional incluindo o farmacêutico clínico na prevenção de complicações hepáticas.

\section{REFERÊNCIAS}

BIGGAR, R. J. AIDS: a global problem. Cancer detection and prevention, v. 12, n. 1-6, p. 169-174, 1988

BRASIL. Ministério da Saúde. Secretaria de Vigilância em Saúde. Boletim Epidemiológico Especial Tuberculose. Brasília (DF): Ministério da Saúde; 2017a.

BRASIL. Ministério da Saúde. Boletim Epidemiológico HIV/AIDS 2019. Brasilia: Ministério da Saúde, 2019.

BARASA, Simon Situma. True story about HIV: theory of viral sequestration and reserve infection. HIV/AIDS (Auckland, NZ), v. 3, p. 125, 2011.

CHURCHYARD, Gavin J. et al. Tuberculosis preventive therapy in the era of HIV infection: overview and research priorities. The Journal of infectious diseases, $v$. 196, n. Supplement_1, p. S52-S62, 2007.

CONDE, Marcus Barreto et al. III Diretrizes para tuberculose da Sociedade Brasileira de Pneumologia e Tisiologia. J. bras. pneumol, v. 35, n. 10, p. 1018-1048, 2009.

DE COCK, Kevin M.; JAFFE, Harold W.; CURRAN, James W. Reflections on 30 years of AIDS. Emerging infectious diseases, v. 17, n. 6, p. 1044, 2011.

DE JONG, Bouke C. et al. Clinical management of tuberculosis in the context of HIV infection. Annu. Rev. Med., v. 55, p. 283-301, 2004. 
DUARTE, Raquel et al. Tratamento da tuberculose latente: Revisão das normas, 2006. Revista Portuguesa de Pneumologia (English Edition), v. 13, n. 3, p. 397418, 2007

EL-SADR, Wafaa M.; TSIOURIS, Simon J. HIV-associated tuberculosis: diagnostic and treatment challenges. In: Seminars in respiratory and critical care medicine. (C) Thieme Medical Publishers, 2008. p. 525-531.

DE FÁTIMA SILVA DE LIMA, Maria. Fatores de risco para hepatotoxicidade do tratamento para tuberculose em pacientes internados e coinfectados pelo HIV. 2009. Dissertação de Mestrado. Universidade Federal de Pernambuco.

HALLAL, Ronaldo et al. O acesso universal ao tratamento antirretroviral no Brasil. Tempus Actas Saude Coletiva, v. 4, n. 2, p. 53-66, 2010.

HORSBURGH JR, C. Robert; RUBIN, Eric J. Latent tuberculosis infection in the United States. New England Journal of Medicine, v. 364, n. 15, p. 1441-1448, 2011.

LALVANI, Ajit; PAREEK, Manish. A 100 year update on diagnosis of tuberculosis infection. British medical bulletin, v. 93, n. 1, p. 69-84, 2009

LEUNG, Chi Chiu et al. Treatment of latent infection with Mycobacterium tuberculosis: update 2010. 2011.

LOBUE, Philip A.; MOSER, Kathleen S. Use of isoniazid for latent tuberculosis infection in a public health clinic. American journal of respiratory and critical care medicine, v. 168, n. 4, p. 443-447, 2003.

MOIR, Susan; CHUN, Tae-Wook; FAUCl, Anthony S. Pathogenic mechanisms of HIV disease. Annual Review of Pathology: Mechanisms of Disease, v. 6, p. 223-248, 2011.

RAMOS, Luiz Roberto et al. Polifarmácia e polimorbidade em idosos no Brasil: um desafio em saúde pública. Revista de Saúde Pública, v. 50, p. 9s, 2016.

RIBAS, Carlise; DE OLIVEIRA, Karla Renata. Perfil dos medicamentos prescritos para idosos em uma Unidade Básica de Saúde do município de ljuí-RS. Revista Brasileira de Geriatria e Gerontologia, v. 17, n. 1, p. 99-114, 2014.

SILVA, J. R. L.; BOÉCHAT, Neio. O ressurgimento da tuberculose e o impacto do estudo da imunopatogenia pulmonar. J Bras Pneumol, v. 30, n. 4, p. 478-84, 2004. 
STERLING, Timothy R.; PHAM, Paul A.; CHAISSON, Richard E. HIV InfectionRelated tuberculosis: Clinical manifestations and treatment. Clinical Infectious Diseases, v. 50, n. Supplement_3, p. S223-S230, 2010.

WORLD HEALTH ORGANIZATION. The Importance of Pharmacovigilance: safety monitoring of medicinal products. World Health Organization. Geneva, 2002.

WORLD HEALTH ORGANIZATION. World report on ageing and health. World Health Organization. Geneva, 2015

[1] Mestre em Ciências Médicas - FMUSP, Especialista em Oncologia Multiprofissional (IEPSL), Especialista em Planejamento Educacional e Docência do Ensino Superior (ESAB), Especialista em Farmácia Hospitalar (FOC), Graduado em Farmácia Bioquímica (USJT).

[2] Doutor em Biotecnologia e Inovação em Saúde. (Anhanguera). Graduação em Farmácia e Bioquímica.

Enviado: Julho, 2020.

Aprovado: Setembro, 2020. 